\title{
Treatment Completed as Prescribed
}

National Cancer Institute

\section{Source}

National Cancer Institute. Treatment Completed as Prescribed. NCI Thesaurus. Code C105740.

Indicates that the patient was fully compliant with the full course of treatment. 